Rodney Oliver J. Aragon,MD'

Samantha Soriano -Castaneda, MD',2,3

Joselito F. David, MD ${ }^{1,2,3}$

1 Department of Otolaryngology Head and Neck Surgery Rizal Medical Center

${ }^{2}$ Department of Otolaryngology Head and Neck Surgery The Medical City

${ }^{3}$ Department of Otolaryngology Head and Neck Surgery Jose Reyes Memorial Medical Center
Correspondence: Rodney Oliver J. Aragon, MD Department of Otolaryngology Head and Neck Surgery Rizal Medical Center

Pasig Blvd., Pasig City 1600

Philippines

Telefax: (632) 6710424

Email: rodneyaragon@hotmail.com

Reprints will not be available from the author.

No funding support was received for this study. The authors signed a disclosure that they have no proprietary or financial interests with any organization that may have direct interest in the subject matter of this manuscript, or in any product used or cited in this report.

Presented at the Interesting Case Contest ( $3^{\text {rd }}$ Place), Philippine Society of Otolaryngology- Head and Neck Surgery Convention, Baguio City April 2007.

\section{Radial Forearm Flap for Tongue Reconstruction}

\author{
ABSTRACT \\ Objective: To present a case of tongue reconstruction using radial forearm free flap.

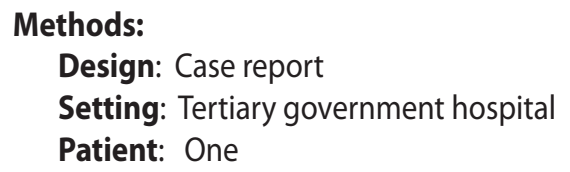

Methods:

Design: Case report

Setting: Tertiary government hospital

Patient: One

Results: A 52-year-old female with a T3N2CM0 Stage IVa right tongue carcinoma underwent tracheotomy, right hemiglossectomy with modified radical neck dissection type III and extended supraomohyoid neck dissection on the left with radial forearm free flap reconstruction. After 1 month, the radial forearm free flap reconstruction in the tongue had acceptable appearance and good tongue mobility with intelligible speech. The patient did not complain about the appearance and function of the left forearm.

Conclusion: The radial forearm free flap is a viable reconstructive option for tongue defects especially where a thin, pliable flap is needed. There is acceptable form and functional restoration with minimal donor site morbidity.

\section{Key words: radial forearm free flap, tongue reconstruction}

A 52-year-old female consulted due to a right lateral tongue mass that was noted 2 months prior as a $1 \times 1 \mathrm{~cm}$ ulcerative lesion with occasional bleeding and tenderness. The patient was a non-smoker. Punch biopsy revealed squamous cell carcinoma, well differentiated and surgery was advised, prompting subsequent admission.

On examination there was a $5 \times 4 \mathrm{~cm}$ right lateral tongue mass involving a portion of the tongue base (Figure 1) with no limitation of tongue movement. There were also a $2 \times 3 \mathrm{~cm}$ slightly fixed level II lymph node on the right side of the neck and a $1 \times 1 \mathrm{~cm}$ movable level lb lymph node on the left side of the neck. Pre-operative staging was T3N2CMO Stage IVa. Of the treatment options recommended, the patient chose surgery with post-operative chemoradiotherapy. 
The patient underwent hemiglossectomy with a portion of the base of the tongue excised for adequate margins. (Figure 2) The neck dissection was done simultaneous with the harvesting of the radial forearm free flap (RFFF). A level lla lymph node on the right side of the neck was noted to have extracapsular spread which was adherent to the anterior scalene muscle.

The cutaneous portion of the RFFF measured $7 \times 5 \mathrm{~cm}$ along with additional $4 \times 5 \mathrm{~cm}$ ofsubcutaneous tissue. (Figure 3 ) The donor site was closed with a split thickness skin graft (STSG). The flap was folded to simulate the tongue shape and part of the flap replaced the floor of the mouth. The harvested subcutaneous tissue was tucked in under the flap to add bulk to the tongue base portion of the flap. (Figure 4) The radial artery was anastomosed to the superior thyroid artery while the cephalic vein was anastomosed to the facial vein. The total length of the operation was 11 hours and 5 minutes.

The patient was decannulated on the $2^{\text {nd }}$ post-operative day and was started on sterile water on the $6^{\text {th }}$ post-operative day. The nasogastric tube was removed on the $7^{\text {th }}$ post-operative day and she was discharged on the $10^{\text {th }}$ postoperative day. Final histopathology revealed tongue SCCA right, with tumor free margins of resection; metastasis 3 of 32 lymph nodes $R$ (levels 2 and 3); negative tumor involvement, left lymph nodes.

Concurrent chemo-radiation therapy post-operation was delayed due to financial difficulties. Four weeks after the operation, there was note of tumor growth at level II on the right side of the neck. (Figure 5) The RFFF was noted to have good color and the patient had good tongue mobility. (Figure 6) The patient was on soft diet since she was edentulous. Speech was intelligible. The patient did not complain about the appearance or function of the left forearm.

The patient subsequently underwent 60 Grays of external beam radiation at $2 \mathrm{G}$ per day at standard fractionation for 30 days at 1 month and 20 days post-operation with erratic compliance. She was asked to extend her radiotherapy for 10 more days but this was not completed due to her demise at 3 months and 23 days post-operation.

\section{DISCUSSION}

Head and neck oncologists are often confronted with the difficult challenge of balancing cancercure and patient survival with preservation of function, cosmesis and quality of life when recommending the best treatment option for the patient. This is difficult in the management of tongue cancer because the tongue is intimately involved in speech and swallowing. With the introduction of microsurgical free tissue transfer, the range of tissues that can be transferred for reconstruction of oral cavity defects has dramatically increased. The radial forearm free flap (RFFF) has emerged as the option of choice among reconstructive surgeons for complex head and neck defects after ablative surgery. Ease of harvest with two synchronous operative teams, potential for sensory innervation, the thin and pliable skin and fascia obtained and the pedicle length and caliber of the vessels are among the reasons for the RFFF's popularity.'

Meaningful statements about cause and effect relationships between reconstructive techniques and function cannot be made

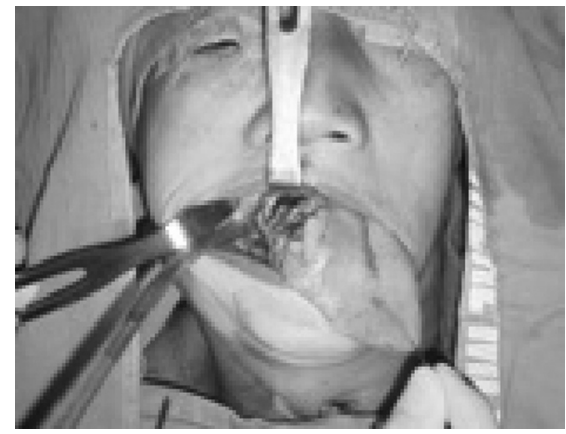

Figure 1. Lesion on the right lateral aspect of the tongue
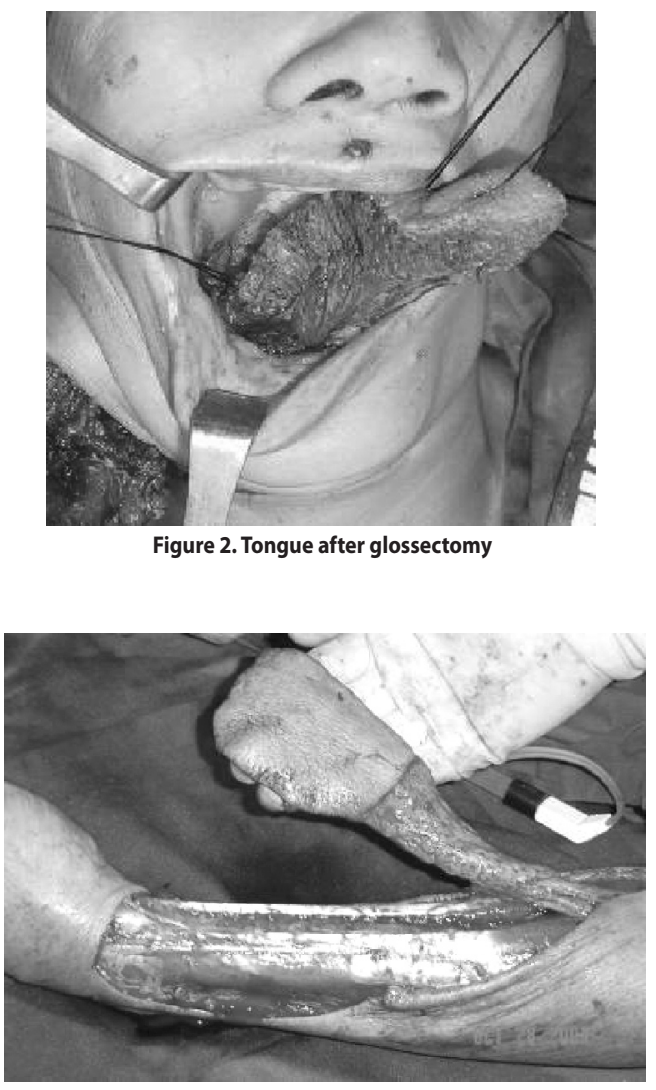

Figure 3. Radial forearm flap.

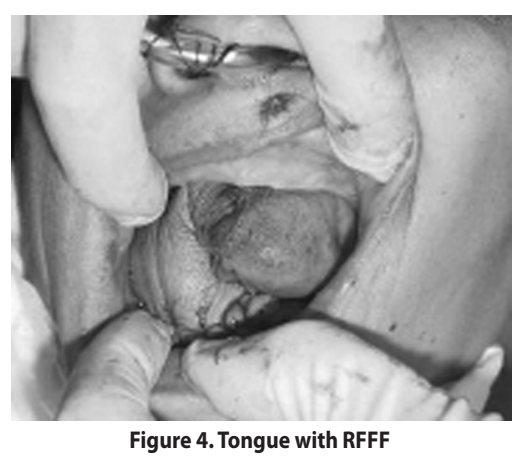

Figure 4. Tongue with RFFF 


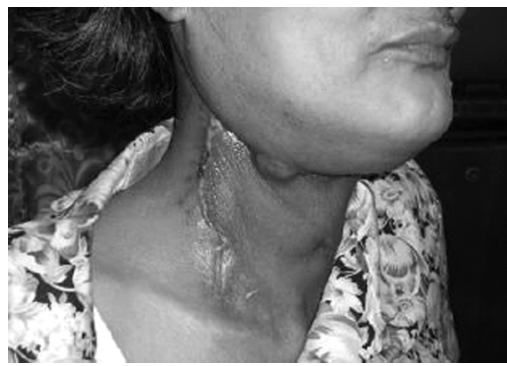

Figure 5. Right side of neck with note of neck node at level II

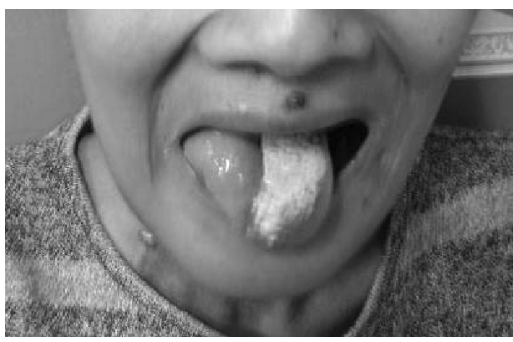

Figure 6. Recomstructed tongue using the radial forearm free flap at 4 weeks post-operation.

without accounting for the site and extent of the surgical resection and many other factors related to the tumor and the patient. There is also need for development of standardized procedures for evaluating functional outcome. ${ }^{2}$ Haughey et al classified tongue defects of the tongue as hemiglossectomy, three quarter glossectomy, total oral glossectomy and base of tongue defects. They advocated the longitudinal fold technique for hemiglossectomy defects. A small amount of overcorrection about (30\%) is needed to allow for decrease in volume of the flap. ${ }^{3}$ This is the technique that was used on our patient to allow for closure of the floor of the mouth defect but at the same time simulating normal tongue appearance with a thin flap. This was evident in the acceptable appearance of the reconstructed tongue, good tongue mobility with intelligible speech and good deglutition of the patient.

Donor site morbidity is another factor that must be considered in the patient about the different reconstruction options. A prospective study on long term functional morbidity of the RFFF donor site revealed 32\% reduced radial nerve sensation, $14 \%$ cold intolerance, $14 \%$ restriction of wrist movement and $28 \%$ poor aesthetic appearance. ${ }^{4}$ This was also confirmed by Toschka et al who opined that it was of no clinical relevance. In their study, $85.7 \%$ of patients displayed optimal functional hand testing values (80-100\%), and $88.6 \%$ gave a positive subjective assessment (80-100\%) of postoperative versus preoperative hand function. ${ }^{2}$ This was evident in our patient who had slight limitation of wrist movement which did not hamper her everyday activities.

The reconstruction is fruitless if there is tumor residual or recurrence. Inspite of advances in surgical ablation and reconstruction, the overall survival of patients with advanced head and neck malignancies has not significantly increased. Thawley and others list the over-all survival of stage IVa tongue carcinoma at only $34 \% .{ }^{5}$ In the National Comprehensive Cancer Network Clinical Guidelines in Oncology, the management for tongue carcinoma in this stage is excision of the primary with comprehensive bilateral neck dissection and concurrent chemoradiotherapy if the nodes have adverse features such as presence of extracapsular spread. ${ }^{6}$ Although this would have been ideal for our case, financial constraints led to delays in adjuvant therapy and limitation to radiotherapy only instead of concurrent chemo-radiotherapy. This may have led to the uncontrolled growth of the regional metastasis and eventual demise of the patient. This is a common plight of patients in the Philippines wherein much needed health care is not within the means of most of the population.

The limitation of the study is that we presented only a single case of tongue reconstruction. An objective assessment of the functional capability of the reconstruction such as videofluoroscopy or a functional endoscopic evaluation of swallowing is the ideal but was not done due to financial and instrumentation constraints. A study comparing different reconstructive techniques for glossectomy defects matched by patient factors and extent of defect based on a standardized functional outcome measure should be done to fully determine the best reconstructive option for glossectomy defects.

The radial forearm free flap is a viable reconstructive option for tongue defects especially where a thin, pliable flap is needed. There is acceptable form and functional restoration with minimal donor site morbidity.

\section{ACKNOWLEDGEMENT}

We would like to thank Dr. Erasmo D.V. Llanes who provided general support and served as our scientific adviser.

\section{REFERENCES}

1. Brown JS. T2 tongue: reconstruction of the surgical defect. Br J Oral Maxillofacial Surg. 1999;37:194-199.

2. Toschka $H$, Feifel $H$, Erli HJ, Minkenberg R, Paar $O$ and Riediger D. Aesthetic and functional results of harvesting radial forearm flap, especially with regard to hand function. Intern J Oral Maxillofacial Surg. 2001; 30: 42-48.

3. Skoner JM, Bascom DA, Cohen Jl, Andersen PE and Wax MK. Short-Term Functional Donor Site Morbidity After Radial Forearm Fasciocutaneous Free Flap Harvest. Laryngoscope. 2003;113(Dec):2091-2094.

4. Richardson D, Fisher SE, Vaughan ED and Brown JS. Radial Forearm Flap Donor-Site Complications and Morbidity: A Prospective Study. Plast Reconstr Surg.1997; 99(1):109-115.

5. Thawley SE, Panje WR, Batsakis JG and Lindberg RD. Comprehensive Management of Head and Neck Tumors. 1999. 34:691-692

6. National Comprehensive Cancer Network Clinical Practice Guidelines in Oncology - Head and Neck Cancers version 2006 